\title{
VALORACIÓN ECONÓMICA DE LOS HOGARES POR LA CALIDAD DEL AIRE EN FLORENCIA, CAQUETÁ
}

Economic valuation of air quality in Florencia, Caquetá

\section{Yelly Yamparli Pardo Rozo1 \\ (ID) https://orcid.org/0000-0001-8060-0281 \\ Jhon Jairo Beltrán Chica ${ }^{3}$ \\ (iD) https://orcid.org/0000-0001-7382-6266 \\ Evelía Sabí Ramirez ${ }^{2}$ \\ https://orcid.org/0000-0002-1626-812X \\ Francy Becerra Torres ${ }^{4}$ \\ https://orcid.org/0000-0002-4725-3692}

\begin{abstract}
${ }^{1}$ Estudiante Doctorado en Ciencias Naturales y Desarrollo Sustentable. Docente, Universidad de la Amazonia. ${ }^{2}$ Magíster en Tributación. Especialista en Gerencia Tributaria. Docente, Universidad de la Amazonia. ${ }^{3}$ Estudiante de Doctorado en Gestión. Especialista en Revisoría Fiscal. Docente, Universidad de la Amazonia. ${ }^{4}$ Magíster. en Tributación. Especialista en Gerencia Tributaria. Graduada Universidad de la Amazonia.
\end{abstract}

Recibido: 25 de octubre de 2019 // Aceptado: 10 de enero de 2020 // Publicado: 30 de enero de 2020

\section{Resumen}

Mejorar la calidad del aire en las ciudades es una de las metas mundiales en los objetivos de desarrollo sostenible, debido a la contaminación atmosférica por la emisión de los gases de efecto invernadero GEI, que en zonas urbanas ha sido atribuible a fuentes fijas como lo son zonas industriales en las ciudades y a fuentes móviles como lo es el flujo vehicular. Florencia en el departamento del Caquetá ha sido considerada como una ciudad intermedia en donde en la última década ha aumentado considerablemente el sector de la construcción y el flujo vehicular, lo que supone un aumento en la emisión de dióxido de carbono por combustión vehicular y material particulado suspendido. Esta investigación halló la disponibilidad a pagar de los ciudadanos por la calidad del aire, a partir del uso de métodos de valoración económica. Se estimó una "Disponibilidad a Recibir Total" (DAR Total) de \$134.150 por hogar al año, la cual está relacionada con la conservación de la salud de los ciudadanos atribuible a la calidad del aire.

\section{Palabras clave}

Contaminación; pagos, calidad del aire, valoración económica.

\begin{abstract}
Improving air quality in cities is one of the global goals in the objectives of sustainable development due to air pollution by the emission of greenhouse gases GHG, which in urban areas has been attributable to fixed sources such as industrial zones in cities and mobile sources such as vehicular flow. Florencia in the department of Caquetá has been considered as an intermediate city, where in the last decade the construction sector and the vehicular flow have increased considerably, which means an increase in the emission of Carbon dioxide, by vehicular combustion and suspended particulate material. This investigation found the availability to pay of the citizens for the quality of the air, from the use of methods of economic valuation. Total willingness to receive (Total DAR) of $\$ 134,150$ per household per year was estimated, which is related to the conservation of citizens' health attributable to air quality.
\end{abstract}

\section{Key words:}

Pollution; Payments, air quality, economic valuation 


\section{Introducción}

El cambio climático es el resultado del impacto antrópico en la atmósfera a consecuencia de las formas de producción y de consumo, que afectó la resiliencia del planeta en la regulación natural de su temperatura (Field, et al,. 2003). El calentamiento global es el principal problema que enfrenta el mundo para el actual y siguiente milenio, que proyecta desastres naturales que afectarán la vida y la economía. El problema surge a partir de la emisión de gases de efecto invernadero (GEI) provenientes de la industria del combustible fósil, la deforestación y la contaminación de los océanos. El cambio climático y las ciudades ecológicas o ecociudades, forman parte de los objetivos de desarrollo sostenible para las metas establecidas para 2030 (Naciones Unidas, 2015).

La calidad del aire, además de considerarse como un principio y derecho en Colombia de acuerdo con la Ley 991993 y la Carta Constitucional, es una externalidad positiva cuyo valor económico inicialmente podría considerarse inexistente debido a que en la mayoría de las regiones el país, la calidad del aire en las zonas urbanas todavía no afecta directamente la salud de las personas, y por ello es complejo atribuir su verdadero valor social. Sin embargo, prescindir de su calidad, se convierte en un problema que trasciende el panorama económico, la salud y el bienestar del ser humano.

La contaminación del aire implica costos para las generaciones presentes, pues una menor disponibilidad de aire limpio, afecta la salud de los individuos, situación que puede traducirse en disminución de la calidad de vida por enfermedades y los costos en dinero y tiempo que se derivan de los pagos por tratamiento, control y prevención de enfermedades. De la misma forma, las futuras generaciones se afectarán mucho más por el cambio climático, si las estrategias actuales no logran mitigar el fenómeno (Aedo, 2005).

Las acciones que priman son: evitar las emisiones y aumentar los sumideros de carbono. En las ciudades, las políticas ambientales buscan frenar las emisiones a partir de incentivos económicos y mecanismos de control de emisiones de combustible fósiles y sus equivalentes para zonas industriales y fluido vehicular. A la industria se le responsabiliza de la emisión del 80 \% de los GEI en el mundo. En la zona rural, la estrategia es la conservación de bosques, la forestación, reforestación y detener la tala indiscriminada; al campo se le atribuye hasta un $20 \%$ de las emisiones de los GEI. Sin embargo, en Colombia el seguimiento y cumplimiento de las normas son de alto costo y demandan una cultura ambiental y de infraestructura que apoyen las orientaciones de la gestión ambiental.

La emisión de GEI en Florencia, por fuentes móviles, específicamente flujo vehicular en la zona urbana, de acuerdo con Becerra (2017) ha crecido significativamente; en cerca de tres años, el parque automotor incrementó en un 34,16\%; pues en el 2012 se contaba con cerca de 34.000 vehículos (automóviles, motos y de carga pesada) y pasó a 42.933 en 2015, que de acuerdo con los consumos de Gasolina Motor, se prevé una emisión desde 106 hasta 137,6 millones de toneladas de dióxido de carbono (CO2) equivalente por año. El 90\% del parque automotor en Florencia lo conforman las motos y el 10\% los automóviles. Además de la contaminación química que genera el parque automotor por la emisión de GEI, también se debe tener en cuenta la contaminación auditiva y visual: el ruido y las partículas suspendidas respectivamente y sus efectos a la salud humana.

Por lo anterior, las preguntas de investigación fueron: ¿Cuáles son los beneficios económicos que perciben los hogares en Florencia por la conservación de la calidad del aire que garantice la conservación de la salud? Y desde la perspectiva de los ciudadanos, ¿cómo impacta la contaminación atmosférica por fluido vehicular en la salud y en la economía de los ciudadanos? Por ello, el objetivo de la investigación fue identificar la relación entre calidad del aire y el estatus de salud de los hogares, y luego, la estimación de una medida que valore el bienestar que genera conservar el aire puro en Florencia. Este estudio es una actualización del realizado por Pardo, Andrade y Lozada en 2015, para 
observar si existe una evolución en el pensamiento y cultura ambiental de la ciudadanía. Se empleó análisis estadístico descriptivo e inferencial; para la primera pregunta se estimó la "Disponibilidad a pagar Total" en la ciudad de Florencia, por evitar la contaminación atmosférica por el flujo vehicular en la zona urbana, como medida económica de bienestar, para lo cual se empleó un modelo de probabilidad lineal. Para la segunda pregunta se empleó un modelo de función de salud, para obtener el valor marginal del daño en la salud generado por la contaminación atmosférica de fuentes vehiculares.

El estudio buscó contribuir con las reflexiones frente a la importancia de las políticas urbanas en materia de regulación vehicular y sus repercusiones en el corto y largo plazo en la salud, bienestar y el cambio climático; de la misma manera, evidencia los beneficios que traería a la comunidad una política que garantice la conservación de la calidad del aíre.

\section{Fundamento teórico}

\section{La atmósfera, la contaminación y la calidad del aíre.}

La composición química de la atmósfera ha evolucionado a lo largo de la historia de la tierra hasta alcanzar la situación actual; actúa como filtro de radiaciones nocivas para la vida, controla la temperatura de la superficie de la tierra, y es fuente de gases que como el oxígeno y el nitrógeno son esenciales para la vida. Su alteración por efecto de la contaminación afecta el delicado equilibrio dentro del cual se desarrolla la vida. La atmósfera tiene un espesor aproximado de 200 kilómetros. De acuerdo con sus propiedades térmicas, la atmósfera se divide en cuatro estratos: troposfera: de 0 a $10 \mathrm{~km}$. desde la superficie de la tierra; la estratosfera: de 10 a $50 \mathrm{~km}$; mesosfera: de 50 a $90 \mathrm{~km}$. desde la superficie de la tierra y la termosfera: sobre los $90 \mathrm{~km}$ (Aranguez, et al., 1999).

El aire es la mezcla de gases que constituye la atmósfera, donde los principales que la componen son: el nitrógeno $(\mathrm{N})$, el oxígeno $\left(\mathrm{O}_{2}\right)$, el argón (Ar) y el dióxido de carbono $\left(\mathrm{CO}_{2}\right)$. Además contiene en menores concentraciones: neón $(\mathrm{Ne})$, el helio $(\mathrm{He})$, el metano $\left(\mathrm{CH}_{4}\right)$, el kriptón $(\mathrm{Kr})$, el hidrógeno $\left(\mathrm{H}_{2}\right)$, el óxido nitroso $\left(\mathrm{N}_{2} \mathrm{O}\right)$, el monóxido de carbono $(\mathrm{CO})$, el xenón $(\mathrm{Xe})$, el ozono $\left(\mathrm{O}_{3}\right)$, el amoníaco $\left(\mathrm{NH}_{4}\right)$ y el óxido de azufre (SO), entre otros.

Según el Ministerio de Ambiente, la contaminación atmosférica es el fenómeno de acumulación o de concentración de contaminantes, entendidos estos como fenómenos físicos o sustancias o elementos en estado sólido, líquido o gaseoso, causantes de efectos adversos al medio ambiente, los recursos naturales renovables y la salud humana que, solos o en combinación, o como productos de reacción, se emiten al aire como resultado de actividades humanas, de causas naturales o de una combinación de estas; aunado a este concepto aparecen los gases de efecto invernadero (GEI) que son los componentes gaseosos de la atmósfera, tanto naturales como antropógenos, que absorben y emiten radiación en determinadas longitudes de onda del espectro de radiación infrarroja emitido por la superficie de la Tierra, la atmósfera y las nubes.

Las fuentes de contaminación atmosférica pueden clasificarse como fijas y móviles, como lo son las chimeneas y el flujo vehicular respectivamente. También existen fuentes naturales como el polvo gracias a la acción de los vientos y lluvias. De acuerdo con el impacto causado, se puede hablar de impacto global, regional, nacional o local. De acuerdo con la naturaleza el contaminante del aire o de la atmósfera puede ser: químico, físico y biológico. Aunque la mayoría de los contaminantes químicos atmosféricos están presentes en la atmósfera, es el cambio en las concentraciones normales lo que crea un evento de contaminación (Ballester, 2005).

Los contaminantes químicos se pueden clasificar en primarios y secundarios. Los primarios son los emitidos directamente a la atmósfera, en tanto que los secundarios son los que resultan de reacciones de oxidación fotoquímica o hidrólisis entre los componentes del aire y los contaminantes primarios. Entre los contaminantes primarios se encuentran: el monóxido de carbono (CO), los hidrocarburos, $(\mathrm{CH})$ y otros compuestos volátiles de carbono (VOC's), óxidos de azufre (SOx), óxidos de nitrógeno (NO), material 
particulado y plomo $(\mathrm{Pb})$. Entre los contaminantes secundarios se encuentran: el dióxido de nitrógeno (NO), los oxidantes fotoquímicos (incluido el ozono), y las deposiciones ácidas (Wark \& Warmer, 1994).

Algunos de los efectos de la contaminación química del aire pueden ser percibidos por las personas de inmediato: malos olores, mareo, dolor de cabeza, etc. Otros efectos, como los cancerígenos, pueden manifestarse después de mucho tiempo. Además de sus efectos sobre la salud humana, los contaminantes del aire afectan a los ecosistemas acuáticos y terrestres, a los cultivos y a la fauna.

Adicionalmente, la contaminación por $\mathrm{O}_{2}$, afecta el desarrollo, crecimiento (afecta la fotosíntesis), producción y reproducción de algunos cultivos como el maíz, el trigo y la soya, y disminuye su capacidad para tolerar ataques de hongos, bacterias, virus e insectos. $\mathrm{Su}$ presencia se ha asociado con daños a ecosistemas boscosos (Cohen et al., 2003).

La atmósfera también puede sufrir contaminación física, la cual puede clasificarse en: contaminación visual, auditiva o por material particulado, que si bien no cambia la composición química del aíre, pero sí afecta a los seres vivos. El ruido es una forma especial de contaminación atmosférica. Es típicamente el ejemplo extremo del contaminante no acumulativo, ya que su efecto se interrumpe una vez que su fuente es controlada. El ruido tiene efectos psicológicos sobre las personas, causando irritabilidad y cansancio. También puede lesionar los órganos del oído y causar sordera. El ruido es además un agente que perturba el normal desarrollo de las especies de fauna silvestre. De otro lado también se encuentra la contaminación visual atribuible a los sólidos suspendidos o a los efectos que causan los paisajes muy intervenidos por publicidad, basuras, etc. (Tyler, et al., 2013).

Impactos y estrategias para mitigar la contaminación atmosférica.

Las estrategias obedecen a la naturaleza de la fuente; entre las principales medidas para mitigar los problemas de las emisiones de contaminantes químicos se tienen las siguientes (Gallego, et al, 2012; Orozco,
Gandur y Londoño, 2006):

-Reformulación de los combustibles.

-Combustibles alternativos a la gasolina y el diesel.

-Los precios de los combustibles.

-Reposición de los vehículos.

-Tecnologías de combustión y el uso eficiente de los combustibles.

-Mantenimiento de los motores.

-Convertidores catalíticos.

-Políticas de manejo de tráfico.

-Transporte público.

-Ordenamiento urbano.

-Sistemas alternativos de transporte.

De otro lado, las decisiones sobre el control de la contaminación por fuentes fijas dependen fundamentalmente de consideraciones de tipo económico y tecnológico. Entre las medidas para mitigar los problemas de las emisiones causados por fuentes fijas se puede mencionar el ordenamiento urbano, pues las industrias deben estar alejadas de las zonas residenciales, recreativas, educativas, etc., de manera que el impacto de las emisiones sobre la salud de la población sea menor; sin embargo, con frecuencia se encuentran industrias en vecindarios residenciales. La concentración de las industrias facilita además la implementación de instrumentos económicos como los permisos mercadeables de emisión, de los cuáles hablaremos posteriormente (Carbal, 2009).

El impacto local de la contaminación atmosférica depende de los gases emitidos, su cantidad, sus fuentes (fijas o móviles), la topografía del área y las condiciones meteorológicas (principalmente la velocidad y dirección del viento, la estabilidad de la atmósfera, la precipitación, la temperatura y la radiación solar). Estas variables determinan los flujos del aire y la dispersión de los contaminantes (Tobón, Sánchezy Cárdenas, 2006).

En atmósferas como la de Ciudad de México y Santiago de Chile y eventualmente la de Bogotá, se crean condiciones en los que los gases contaminantes tienden a acumularse cerca de la superficie (se comportan como contaminantes acumulativos). Lo contrario ocurre en aquellos sitios donde la atmósfera es inestable. Bajo condiciones de inestabilidad, el aire 
cálido de la superficie fluye hacia arriba y arrastra los contaminantes hacia alturas donde no afectan la salud humana.

En consecuencia, la capacidad de la atmósfera de facilitar o impedir el movimiento vertical de las masas de aire, afecta la concentración de los contaminantes cerca de la superficie que es donde se desarrolla la vida humana. Otros factores que determinan los niveles de acumulación de contaminantes en la atmósfera son la topografía y la distribución espacial de las ciudades. La topografía afecta el movimiento y la dispersión de los contaminantes al afectar la dirección y velocidad del viento. Por ejemplo, en Ciudad de México, además de los problemas de inversión térmica, los contaminantes se acumulan en los estratos bajos de la atmósfera porque la ciudad está en un valle rodeado de montañas que limitan el flujo del viento. Adicionalmente, en zonas con edificios altos, se crea un efecto (street canyon effect) que impide el flujo vertical del aire.

En el caso de la Ciudad de México, por su altura sobre el nivel del mar $(2240 \mathrm{~m})$, la producción de "smog" fotoquímico es mayor, además existe un problema de ordenamiento urbano, pues durante la mayor parte del año los vientos soplan desde la zona industrial hacia las zonas residenciales, arrastrando hacia ellas la contaminación.

Los principales fenómenos asociados a los daños en la atmósfera son: el calentamiento global, la inversión térmica y la lluvia ácida.

La contaminación genera impactos en la salud de los humanos, daños en especies de flora y fauna y los ecosistemas, alterando el equilibrio y el bienestar de las formas de vida. Impactos en las vías respiratorias, estrés auditivo y visual, son algunos de los efectos que pueden conducir a la muerte y a la morbilidad (Simioni, 2003).

Entre las externalidades negativas por contaminación atmosférica se destaca la del uso del automóvil. Se estima que el transporte por carretera genera casi el $15 \%$ de las emisiones totales de $\mathrm{CO}_{2}$, el principal gas responsable del efecto invernadero y del calentamiento global del planeta. Además, y sobre todo en el caso de los vehículos que incorporan un motor diésel, son en gran parte los responsables de la llamada contaminación atmosférica local, directamente vinculada a las emisiones de ciertos gases y partículas sólidas que generan graves problemas de salud. Recientemente, la Comisión Europea hizo público que, según sus estimaciones, estas emisiones locales son las responsables, de una manera u otra, de unas 360.000 mil muertes al año en la UE, es decir, siete veces el número que causan los accidentes de tráfico. (Espasa et al., 2006).

\section{Estudios sobre contaminación atmosférica y sus impactos.}

Dockery y Pope (1994) comparan estudios recientes que demuestran evidencias de aumento de la mortalidad y la morbilidad asociadas a concentración moderada de partículas suspendidas. En estudios realizados en Estados Unidos y Europa (Barnes, et al., 1994; Peters, 1996), se demostró que la concentración de la media anual de las partículas totales estuvo significativamente asociada con la prevalencia de tos y bronquitis en niños escolares, y fue más fuerte en aquellos niños con diagnóstico de asma. Otra evidencia describe que niños de 5 a 7 años de Ciudad de México con asma moderada son afectados por concentraciones de partículas suspendidas menores de $10 \mathrm{U}$ y en partículas de 2,5 U, en el cual se demostró una fuerte asociación entre los niveles de Material Particulado (PM) y el flujo respiratorio máximo, mientras que los síntomas respiratorios fueron asociados tanto a PM10 como a ozono (RomieuI, et al., 1995; 141:546-53). En estudios realizados en Chile se reporta el incremento de enfermedades respiratorias agudas asociado fuertemente con los niveles de partículas en suspensión Pm10 (Peters et al., 1996).

En Colombia se han realizado diferentes estudios para tratar el tema de la calidad del aire, como en Franco et al., (2016) donde se concluye la importancia de involucrar la variable calidad del aire al momento de diseñar infraestructuras orientadas a modos de transporte no motorizado; en el estudio de Sánchez et al., (2013) la concentración de los gases de efecto invernadero en Cartagena, se encuentran dentro de los límites recomendados por las normas nacionales, pero las pequeñas partículas mantienen una alta 
concentración, especialmente en las zonas cercanas a carreteras con alto tráfico vehicular.

Es necesario evaluar el impacto de las partículas en los procesos respiratorios de las personas que viven cerca de carreteras; en la Universidad de Medellín se realizó una estimación de las emisiones de contaminantes atmosféricos provenientes de fuentes móviles en el área urbana de Envigado, (Londoño et al., 2013) en la que se concluye que las mayores emisiones se presentaron sobre las vías con mayor tránsito vehicular; el mayor aporte lo hace el monóxido de carbono (CO) con 18,4 t por día $\mathrm{d}^{-1}(71,3 \%)$. La mayor emisión de este contaminante es a las 12:00 horas con 1,4 $\mathrm{th}^{-1}(7,4 \%)$.

En el departamento del Caquetá se desarrolló un estudio "Efectos socioeconómicos de la contaminación atmosférica por emisiones vehiculares en la ciudad de Florencia Caquetá y su impacto en la salud" realizado por Losada, (2013) quien evidenció la existencia de una estrecha relación entre el estatus de la salud y la exposición al aire contaminado de acuerdo con la percepción del ciudadano en Florencia; además se hizo una recomendación al gobierno y al sector transporte para que lideraran políticas de responsabilidad social empresarial acordes a las condiciones demográficas y tecnológicas que vienen en el futuro por el desarrollo de ciudades intermedias.

De acuerdo a todos estos estudios se ve la necesidad de regular la contaminación atmosférica con políticas ambientales y mecanismos de regulación apropiados al contexto de cada país.

En la experiencia internacional muchos estudios indican que la contaminación atmosférica sí afecta positiva y significativamente tanto la morbilidad como la mortalidad. De acuerdo con Ortiz, Martínez y García (1998), para el caso de Estados Unidos, cita a Lester y Seskin (1973) presentan evidencia estadística significativa de que los contaminantes en el aire pueden iniciar o agudizar un amplio rango de enfermedades respiratorias, incluyendo bronquitis crónica, enfisema, asma y posiblemente cáncer de pulmón. El estudio concluye que la reducción de estos contaminantes disminuye significativamente la tasa de mortalidad.
Gerkin y Stanley (1986) avanzan sobre el trabajo anterior, utilizando una metodología basada en la revelación de la disponibilidad a pagar de los habitantes de Saint Louis (USA) por un mejoramiento de la calidad del aire. Los costos incurridos por el tratamiento médico de enfermedades respiratorias son incorporados en una función de producción de salud que presenta argumentos en dichos gastos, la calidad del aire y una serie de argumentos cualitativos -salud, educación, raza, etc.-. El estudio concluye que las personas analizadas estarían dispuestas a pagar 25 dólares anuales -el 60\% del valor de una consulta médica- para disfrutar una disminución del 30\% en los niveles de contaminación por ozono en el área de St. Louis.

De otro lado en los estudios de Ostro (1983, 1987), en los que se emplea el enfoque de la función dosisrespuesta (ver marco metodológico) se demuestra de forma robusta y significativa la correlación positiva entre la contaminación atmosférica por la concentración de material particulado y dióxido de azufre con la morbilidad de la población en 84 ciudades norteamericanas. El análisis econométrico controló las características socioeconómicas de la población afectada y las características atmosféricas de las ciudades de la muestra. También Ostro et al. (1995), utilizando información sobre niveles de contaminación por material particulado (PM10) y mortalidad en Santiago de Chile, encuentra evidencia robusta sobre el efecto de esta contaminación sobre la tasa de mortalidad.

En Colombia son variados los estudios que buscan analizar la relación entre contaminación atmosférica y enfermedades o repercusiones en la salud humana. Se destaca el estudio de Ortiz, Escobar y García (1998), el cual presentan brevemente algunos trabajos científicos sobre la relación entre contaminación y salud en Cali y los municipios aledaños de Yumbo y Jamundí. Rico (1995) realiza un análisis comparativo entre la población residente en Yumbo -zona de alta contaminación atmosférica- y la población residente en Jamundí, la cual se caracteriza por bajos niveles de contaminación atmosférica. La evidencia estadística sugiere que la incidencia de enfermedades respiratorias 
es significativamente mayor en Yumbo que en Jamundí. Además, el estudio también encuentra evidencia significativa de que la contaminación atmosférica incide positivamente en la demanda de servicios médicos por la comunidad. La experiencia en el estudio en Florencia, Caquetá, en 2015 en Pardo, Andrade y Losada (2015), ya había demostrado cómo el fenómeno de contaminación atmosférica ya estaba relacionado con la emisión por fluido vehicular.

\section{Métodos}

La investigación aplicada buscó desarrollar y replicar experiencias tomadas de otros sectores y adaptadas a un contexto determinado. Se realizó un análisis descriptivo de variables cualitativas y cuantitativas además de un análisis paramétrico apoyado en la econometría y los métodos de valoración económica ambiental. La investigación también es de naturaleza cuantitativa pues intenta validar resultados a partir de la estadística descriptiva y la estadística inferencial para proyectar el comportamiento de variables socioeconómicas. Contempla el análisis de variables discretas, de tipo categórico y ordenativo que describen atributos, y de tipo continuo para tratar datos numéricos. Según su función, la investigación es de naturaleza positiva, ya que pretende describir los fenómenos como son (Mason y Marshall, 2000).

La población objeto de estudio son los hogares de la ciudad de Florencia, y para la determinación del tamaño de la muestra de estudio de acuerdo con Jany (1994), se realizó mediante el muestreo aleatorio simple, para población finita. Los parámetros estadísticos para calcular la muestra fueron:

a: La precisión o error $5 \%$.

Z: El nivel de confianza en el tamaño de la muestra $(1,96)$.

S: La variabilidad $(0,44)$.

$\mathrm{N}$ : Población (se tiene que la población está conformada por los 160.000 habitantes de los cuales el 96\% vive en la zona urbana, es decir 153.600).

Para el estudio realizado se tomó un nivel de confianza del 95\% por consiguiente utilizando las tablas para distribución normal se calculó el valor $\mathrm{Z}$ que corresponde a 1,96 (con aproximación a dos decimales); se consideró la variabilidad (S) igual a 0.5 dado que no hay antecedentes sobre la investigación. De igual forma se calculó la muestra para los participantes directos con un $\mathrm{N}$ de 153.600. La fórmula usada para determinar la muestra es la siguiente. Este estudio se apoyó en la base de datos de Becerra (2017).

$$
\mathrm{n}=\frac{\mathrm{Z}^{2} \mathrm{~S}^{2} \mathrm{~N}}{\mathrm{N \alpha ^{2 } + ( Z S ^ { 2 } )}}=376
$$

Ecuación 1. Formula para muestreo

Para la recolección de la información directa se empleó la técnica de encuesta, la cual fue dirigida a los jefes de hogar, a través de preguntas abiertas y cerradas que incluyó varios bloques de información. El primer bloque se realizó referente a los aspectos socioeconómicos de los encuestados (género, edad, ingresos, situación laboral, personas en el hogar, estrato, etc.). El segundo bloque, trató sobre los hábitos frente al tratamiento y prevención de las enfermedades respiratorias a consecuencia de la contaminación y las características del sistema de salud al que pertenecen; el tercer bloque hizo referencia a los hábitos relacionados con el servicio de transporte (público y privado) que supone comportamientos, necesidad del servicio, frecuencia y medios para conocer la demanda de vehículos y con ello un proxy a la generación de $\mathrm{CO}_{2}$ como huella ecológica de cada familia por esta actividad. El cuarto bloque de preguntas, corresponde al caso hipotético en el que se desea conocer la disponibilidad a pagar a la que respondería la comunidad frente al mejoramiento de la calidad en el aire en la ciudad de Florencia. Las variables inmersas en el modelo matemático que se presenta a continuación en los siguientes modelos empíricos, fundamentados en la Valoración Económica Ambiental.

\section{Enfoque de la Valoración económica ambiental}

La economía ambiental trata la relación entre el sistema económico y el ambiente, que según Kolstad (1998) tiene tres objetivos clave: el primero, es reconocer la importancia que tienen los recursos naturales y ambientales para el sistema productivo y de consumo, como motor de la economía; el segundo es evidenciar los impactos del consumo y de la producción en el 
ambiente; y el tercero, es proponer mecanismos que permitan regular las actividades tanto de producción y consumo para armonizar el sistema económico con la capacidad de resiliencia de los ecosistemas. Dentro del segundo objetivo, la valoración económica ambiental (VEA) desarrolla los métodos para valoración de bienes y servicios que son denominados como "no mercadeables", entendidos como aquellos que no poseen un mercado convencional, entre los que se encuentran los bienes y servicios ambientales. La VEA ofrece fundamentos teóricos y herramientas validadas para hallar el valor económico a bienes y servicios ambientales que no cuentan con un sistema de precios para productores y consumidores. A través de los métodos de VEA se pueden hallar medidas de bienestar basadas en la teoría microeconómica, entre ellas, la variación compensada, la disponibilidad a pagar total y marginal y el excedente del consumidor (Pardo, Andrade y Hermosa, 2012).

Para estimar en términos económicos los efectos de los cambios en el ambiente sobre la salud humana, se debe estudiar la relación entre el cambio ambiental y el cambio en el estatus de salud. Para el caso de la contaminación del aire, para medir el estado del recurso, puede establecerse mediante el nivel de partículas suspendidas en el aire, la cantidad de bióxido de carbono, la cantidad de bióxido de azufre o los sólidos suspendidos por volumen. Igualmente, se debe estudiar la relación existente entre el cambio en el estatus de salud y alguna medida monetaria, que permita encontrar el equivalente del cambio en la salud en términos de un esquema de compensación, expresado por medio de una disponibilidad a pagar o una disponibilidad a aceptar (Azqueta, 1995).

Teóricamente para la valoración económica de los impactos de la contaminación ambiental sobre la salud humana existe el supuesto de que las preferencias de los individuos están caracterizadas por la sustituibilidad entre el ingreso y la salud; Kolstad (2001) menciona que la contaminación ambiental que afecta la salud de las personas puede generar pérdidas en el bienestar a partir de los siguientes aspectos:

1. Gastos médicos asociados con los tratamientos a las enfermedades generadas por la contaminación ambiental, incluyendo el costo de oportunidad del tiempo gastado en obtener el tratamiento.

2. Pérdida de Salario por enfermedad.

3. Gastos preventivos asociados a actividades defensivas y de evasión de las enfermedades inducidas por la contaminación.

4. El malestar asociado con los síntomas de la enfermedad y pérdida de oportunidades de disfrute del ocio.

5. Cambios en las expectativas de vida o riesgo prematuro de muerte.

La reducción en los niveles de contaminación beneficia a la sociedad debido a que disminuyen algunos o todos los efectos mencionados y su equivalente monetario sería una medida de los beneficios económicos que experimentarían las personas por mejoras en el ambiente. A continuación se presentan los principales métodos alternativos de valoración ambiental.

\section{Modelo de producción de salud de hogares}

La función de producción de salud relaciona variables exógenas (incluyendo variables ambientales), y de elección (medicina preventiva y costo de tratamiento), para alguna medida del estado de salud. Freeman (1993) menciona que uno de los supuestos del método es que los individuos conocen su función de producción, escogiendo el nivel óptimo de producción y seleccionando los insumos para minimizar los costos relacionados con un determinado nivel de salud. A continuación se presenta el desarrollo del modelo de función de producción de salud presentado por este autor. Se propuso el siguiente modelo 1 para estimar en la muestra, de qué depende el estatus de salud:

$$
\begin{gathered}
S=\beta_{\circ} \pm \beta_{1} V T \pm \beta_{2} V P \pm \beta_{3} Q \\
\text { Ecuación } 2
\end{gathered}
$$

Para ello se establecen las siguientes variables:

Estatus de salud (S): variable dependiente discreta ordenativa, que representa el estatus de salud para el encuestado durante el último año frente a enfermedades respiratorias por contaminación atmosférica en Florencia. Toma valores desde 0 hasta 5. 
0 pésimo, 1 malo, 2 regular, 3 aceptable, 4 bueno, 5 excelente.

Valor tratamiento (VT): variable cuantitativa independiente en pesos colombianos que representa el valor de los costos para tratar una enfermedad respiratoria por contaminación atmosférica.

Prevención (VP): variable cuantitativa independiente en pesos colombianos que representa el valor de los costos para prevenir una enfermedad respiratoria por contaminación atmosférica.

Contaminación $(Q)$ : variable discreta ordenativa independiente, que representa la valoración sobre la percepción del encuestado frente al nivel de exposición de contaminantes atmosféricos. Toma el valor de 0 cuando no existe un nivel de exposición, 1 nivel de exposición bajo, 2 nivel de exposición medio, 3 nivel de exposición alto.

Los signos esperados para las derivadas parciales en la función de salud son:

$\beta_{1}>0$, indicando que a un mayor nivel de contaminación en el aire mayor es el número de días enfermo.

$\beta_{2}<0$, indicando que a un mayor nivel de tratamientos médicos, menor es el número de días enfermo.

$\beta_{3}<0$, indicando que a un mayor nivel de actividades preventivas menor es el número de días enfermo.

La salud se mide entonces por el número de días de enfermedad del individuo. Sin embargo, se presenta el problema que no se tiene en cuenta la gravedad de la enfermedad. Por otro lado, se hace necesario incluir otros determinantes del estado de salud del individuo, como características socioeconómicas del individuo, por ejemplo, edad, sexo, ingresos, educación, entre otras. También se propone el modelo 2 para hallar la disponibilidad a pagar total por un proyecto que garantiza la conservación de la calidad del aire, según Mendieta (2001) y Uribe et al., (2003).

\section{$D A P=\beta_{0} \pm \beta_{1} V A P \pm \beta_{2} N E \pm \beta_{3} G e \pm \beta_{4} I N G \pm \beta_{5} P H+\mu_{i}$} Ecuación 3. Disponibilidad a Pagar
Donde VAP es el valor de la disponibilidad a pagar; NE es el nivel educativo del encuestado; ING es el ingreso mensual; PH es el número de personas en el hogar; $\mu$ : margen de error del modelo

Para el procesamiento de los datos en el análisis inferencial se empleó análisis de regresión, bajo el método de mínimos cuadrados ordinarios, modelo de probabilidad lineal y se emplearon los pasos de la metodología econométrica de acuerdo con Gujarati y Porter (2010), así: Planteamiento teórico; Proposición del modelo matemático; Modelo econométrico; Obtención de datos; Estimación de parámetros; Pruebas de hipótesis para validez estadística y uso del modelo como herramienta de pronóstico.

\section{Resultados y discusión}

Características socioeconómicas de los encuestados La muestra de estudio registró la participación de un $86 \%$ de hombres y un 14\% mujeres; de los encuestados el 73\% son casados; el 19\% viven en unión libre y el porcentaje restante no tienen cónyuge o son solteros; con una edad de 44 años según las medidas de tendencia central. El 100\% tiene personas a cargo. El $86 \%$ tienen formación en posgrado; el $12 \%$ correspondiendo son profesionales y el $2,3 \%$ restante tienen formación en tecnologías. El ingreso laboral promedio fue de $\$ 2.625 .0000$ mensuales, donde las ocupaciones en cargos docentes, trabajadores independientes y comerciantes.

\section{Valoración económica de la calidad del aire}

El 76\% de la muestra ha experimentado contaminación auditiva (en las horas pico) generada por el parque automotor; el 84\% hablan también de contaminación visual por mala disposición de basuras, publicidad y daños en las calles atribuidos a una pésima cultura ciudadana. Otro 5\% de los encuestados proponen incluso la percepción de una contaminación olfativa, por los malos olores de basuras mal depositadas, cañerías asociadas a la ausencia de una cultura ciudadana y mal mantenimiento de alcantarillas.

Se indagó sobre la disponibilidad a pagar por un proyecto hipotético que garantice la calidad del aire de la zona urbana de Florencia, ante el aumento del 
parque automotor, que podría aumentar la probabilidad de contraer enfermedades asociadas: gripe, contaminación visual y en general de las vías respiratorias. Se encontró que un 97,3\% tienen una DAP positiva y diferente de cero $(\$ 0)$, y el $17 \%$ tienen una DAP igual a cero $(\$ 0)$, donde el promedio fue de $\$ 27.763$. Los resultados del modelo de salud se presentan en la Tabla 1.

Luego, el modelo queda así:

$$
\begin{gathered}
S=\beta_{0} \cdot \beta_{1} M_{1} D_{+} \beta_{2} G T G R \pm \mu_{i}=4,26+0,07(M E D)- \\
0,00000278(G R I P E)=4,323
\end{gathered}
$$

El modelo permitió identificar que existe una relación positiva entre el estatus de salud y los gastos médicos; es decir que a mayor inversión en tratamientos médicos y en prevención de enfermedades, la calidad de la salud tiene probabilidades de aumentar. Por otro lado, la relación entre estatus de salud y la percepción de la contaminación del aire es negativa, indicando que entre mayor contaminación del aire el estatus de salud de las personas disminuye. Estos resultados son consistentes con los presentados en Pardo, Andrade y Lozada en 2015. Para hallar la disponibilidad a pagar por un proyecto que garantice la conservación de la calidad del aire en la ciudad arrojó los resultados que se presentan en la Tabla 2.

Tabla 1.

Análisis de regresión modelo 1

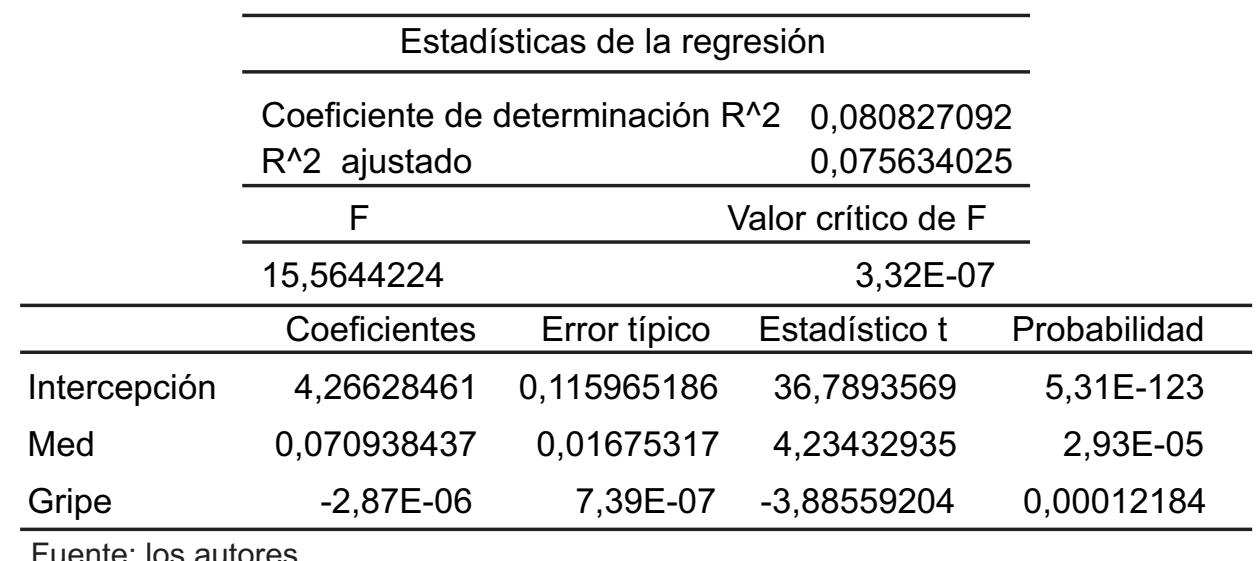

Tabla 2.

\begin{tabular}{|c|c|c|c|c|}
\hline & \multicolumn{3}{|c|}{ Estadísticas de la regresión } & \\
\hline & \multirow{2}{*}{\multicolumn{2}{|c|}{$\begin{array}{l}\text { Coeficiente de correlación múltiple } \\
\text { Coeficiente de determinación } \mathrm{R}^{\wedge} 2\end{array}$}} & 0,60123321 & \\
\hline & & & 0,361481373 & \\
\hline & \multicolumn{3}{|c|}{$\begin{array}{lll}\mathrm{F} & \mathrm{V} \\
\end{array}$} & \\
\hline & \multicolumn{2}{|c|}{29,11500122} & $9,21 \mathrm{E}-32$ & \\
\hline & Coeficientes & Error típico & Estadístico t & Probabilidad \\
\hline Intercepción & 0,757664199 & 0,04830917 & 15,6836513 & $1,29 E-42$ \\
\hline VAP & 6,88E-06 & $7,39 \mathrm{E}-07$ & 9,30888913 & $1,30 \mathrm{E}-18$ \\
\hline $\mathrm{NE}$ & 0,099558042 & 0,02604608 & 3,82238042 & 0,000155693 \\
\hline Genero & $-0,053679681$ & 0,0198034 & $-2,7106295$ & 0,007037163 \\
\hline Ing & $-7,55 \mathrm{E}-08$ & 1,83E-08 & $-4,1358495$ & 4,41E-05 \\
\hline $\mathrm{PH}$ & 0,029475741 & 0,00688784 & 4,27938877 & $2,41 \mathrm{E}-05$ \\
\hline
\end{tabular}

Análisis de regresión modelo 2.

Fuente: los autores 
Se observa que las variables fueron relevantes. El coeficiente VAP fue positivo, indica que a mayor valor de la DAP mayor probabilidad de pago del individuo, lo que es contradictorio con el ingreso, pues a mayor valor del ingreso, disminuye la probabilidad de estar dispuesto a pagar. El signo negativo del género indica que de forma comparativa, los hombres tienen una mayor DAP que las mujeres; y a mayor número de personas en el hogar la probabilidad de estar dispuestos a pagar aumenta. Luego, la ecuación será:

\section{$\mathrm{DAP}=0,76+0,000000688^{*} \mathrm{VAP}+0,095^{*} \mathrm{NE}-$ $0,05^{*}$ GENERO - 0,000000007555*ING + $0,029^{*} \mathrm{PH}=\$ 134.150$.}

Cómo se esperaba un resultado negativo de la DAP pero el resultado fue positivo, se puede concluir que aún no existe un mercado para la calidad de aire, y se percibe una Disponibilidad a Aceptar DAA, es decir, los encuestados consideran que deben ser compensados en lugar de pagar por la calidad del aire. La DAA Total será el valor hallado multiplicado por las 376 personas encuestadas para un total de $\$ 50 ' 440.365$. En el estudio de Becerra (2017), concentra el uso de estos estudios para proponer el diseño de un impuesto y la forma de estimar su base gravable.

Los resultados de Lozada (2014); Pardo, Andrade y Losada (2015) y Becerra (2017) en Florencia, Caquetá, permiten argumentar que aunque la comunidad si bien percibe una relación entre la salud y la contaminación atmosférica, aún no se vislumbra el valor del aire limpio, lo que puede atribuirse a que este aún no constituye un bien escaso; es decir, aún no se percibe un daño ambiental en el aire de la ciudad. Sin embargo, el aumento del parque automotor en Florencia y la fragilidad de la calidad del aire frente a los factores ambientales en la proliferación de agentes patógenos en el contexto tropical, hace necesario evaluar y estudiar estos temas, para ver cómo los ciudadanos valoran las condiciones del entorno, y proponer políticas para fomentar una cultura ciudadana frente a la responsabilidad de conservar el aire limpio y evitar que se llegue a un desarrollo urbano en la Amazonia que pueda afectar el equilibrio y resiliencia del aire en la zona urbana. Otros resultados del impacto de la calidad del aíre en la economía es el estudio de Carriazo (1999), quien demuestra cómo la externalidad puede afectar el precio de las viviendas en el sector urbano en Bogotá.

\section{Conclusiones}

Los ciudadanos perciben los problemas de la contaminación del aíre en la emisión de GEI por el parque automotor y reconocen que algunos problemas de salud pueden atribuirse a la calidad atmosférica, sin embargo en la valoración ambiental aún no existe un valor por conservar el aíre limpio, en cambio se percibe como una disponibilidad a aceptar o a recibir (DAA ó DAR) o una compensación que demuestra que el recurso (Calidad del aire) no es escaso. La DAA total fue de $\$ 50,5$ millones en la muestra, lo que implica que de faltar la calidad del aire, los hogares solicitarían este valor para compensar su bienestar económico.

La contaminación atmosférica es una externalidad negativa, que además de que puede afectar la salud de las personas, genera un costo en medicamentos, tratamientos de recuperación y prevención; costos de transporte, costo de oportunidad del tiempo y su calidad para el disfrute. Esto evidencia que perder la calidad del aire, implica en perder calidad de vida con repercusiones en materia económica y social.

Estudios futuros deberán concentrarse en la propuesta de políticas públicas para evitar la contaminación auditiva, visual, química y biológica del aire en la ciudad. El ordenamiento urbano, el control del crecimiento y actualización del parque automotor, al igual que implementar una cultura de manejo de enfermedades de fácil proliferación asociadas a las condiciones del ambiente, son políticas que pueden diseñarse a partir de estos resultados.

\section{Referencias bibliográficas}

Aedo, C. (2005). Evaluación del impacto. Naciones Unidas, Comisión Económica para América Latina 
y el Caribe.

Aránguez, E., Ordóñez, J. M., Serrano, J., Aragonés, N., Fernández-Patier, R., Gandarillas, (1999). Contaminación atmosférica y su vigilancia, Revista Salud Pública 73:123-132

Asamblea Nacional Constituyente (1991). Constitución Política de la República de Colombia, Bogotá D.C.

Azqueta Oyarzun, D. (1995). Valorización económica de la calidad ambiental, Madrid 1995.

Ballester, F. (2005). Contaminación atmosférica, cambio climático y salud. Rev Esp Salud Pública, 79(2), 159-175.

Barnes P. J. (1994). Air pollution and asthma. Postgraduate medicaljournal, 70(823), 319-325.

Becerra, F. (2017). Estudio de impacto socioeconómico para el diseño de un impuesto verde por emisión de gases contaminantes de vehículos automotores en el departamento del Caquetá (Tesis de Maestría en Tributación). Universidad de la Amazonía, Florencia, Colombia.

Carbal Herrera, A. (2009). La valoración económica de bienes y servicios ambientales como herramienta estratégica. Criterio Libre, 7(10), 71-89.

Carriazo, F. (1999). Impactos de la contaminación del aire en el precio de la vivienda: una valoración económica para Santa fe de Bogotá. (Tesis de Maestría en Economía Ambiental y de Recursos Naturales). Universidad de los Andes, Bogotá D.C. Colombia.

Congreso de la República de Colombia (1993). Ley 99 del 22 de diciembre de 1993 por la cual se crea el Ministerio del Medio Ambiente, se reordena el Sector Público encargado de la gestión y conservación del medio ambiente y los recursos naturales renovables, se organiza el Sistema Nacional Ambiental, SINA y se dictan otras disposiciones. Bogotá D.C.: Congreso de la República de Colombia.

Cohen AJ, Anderson HR, Ostro B, Pandey KD,
Kryzanowsky M, Kuenzly N, et al. (2003). Mortality impacts of Urban Air Pollution. En: Ezzati M, Lopez AD, Rodgers A, Murray CJL (editores). Comparative Quantification of Health Risks: Global and Regional Burden of Disease Attributable to Selected Major Risk Factors. Ginebra: Organización Mundial de la Salud.

Dockery DW, Pope III CA. (1994). Acute respiratory effects of particulate air pollution. Environmental Epidemiology Program. Harvard School of Public Health, Boston, Massachusetts. Annu Rev Public Health. 15:107-32.

Espasa, M., Lladó, A., \& Nadal, M. (2006). Propuesta de reforma de la fiscalidad sobre el automóvil. RACC automóvil club.

Field, B. C., Field, M. K., \& Deocón, G. T. (2003). Economía ambiental. McGraw-Hill.

Freeman, A. (1993). The Measurement of Environmental and Resource Values. Theory and methods. RFF PRESS, Washington, USA.

Gallego, A., González, I., Sánchez, B., Fernández, P., Garcinuño, R., Bravo, J., \& Durand, J. (2012). Contaminación atmosférica. España: UNED Universidad Nacional de Educación a distancia.

Gujarati, D. y Porter, D. (2010). Econometría Básica. 5 ed. McGraw Hill, Santafé de Bogotá.

Gujarati, D. (1999). Econometría Básica. 3 ed. McGraw Hill, Santafé de Bogotá.

Hernández, R., Fernández, C. \& Baptista, L. (2014). Metodología de la Investigación. Quinta Edición, México.

Jany, J. (1994). Investigación integral de mercados, un enfoque operativo. Mc Graw Hill, Colombia.

Kolstad, Ch. (2001). Economía ambiental. Oxford University Press, Mexico.

Londoño, J., Correa, M. A., \& Palacio, C. A. (2013). Estimación de las emisiones de contaminantes 
atmosféricos provenientes de fuentes móviles en el área urbana de envigado, Colombia. Revista EIA, 8(16), 149-162.

Losada, J. W. (2014). Efectos socioeconómicos de la contaminación atmosférica por emisiones vehiculares en la ciudad de Florencia, Caquetá y su impacto en la salud. Universidad de la Amazonia, Florencia, Caquetá.

Mason, R; Lind D, Marshall W. (2000) Estadística para administración y economía. Alfaomega, Bogotá 2000.

Mendieta J. (1999) Manual de Valoración Económica de Bienes no Mercadeables., documento CEDE 10. Universidad de los Andes, Bogotá.

Ministerio de Ambiente, Vivienda y Desarrollo Territorial (2003). Metodologías para la valoración económica de bienes, servicios ambientales y recursos naturales.

Molina, C. M.(s.f.) Instrumentos económicos para enfrentar el cambio climático. S.E.

Naciones Unidas. (2015). Objetivos de Desarrollo Sostenible. Asamblea General, 12 de agosto de 2015. Recuperado en:www.un.org/es/ comun/docs/?symbol $=\mathrm{A} / 69 / \mathrm{L} .85$. NACIONES UNIDAS (2015).

Organización Mundial de la Salud. (2004). Informe de Estado de la salud en el mundo 2002 (en línea) https://www.who.int/whr/2002/chapter4/en/index7.h $\underline{\mathrm{tml}}$

Ortiz, Martínez \& García (1998). Contaminación Atmosférica y Salud. Departamento Administrativo de Gestión del Medio Ambiente (DAGMA) de la ciudad de Cali. Cali, Valle.

Orozco, D. T., Gandur, A. F. S., \& Londoño, M. V. C. (2006). Regulación ambiental sobre la contaminación vehicular en Colombia: ihacia dónde vamos? Medellín. Universidad de Antioquia-CIE.

Pardo, Y.Y; Andrade, M.C y Losada, JW (2015). Impacto en la salud por contaminación atmosférica (emisiones vehiculares en la ciudad de Florencia, Caquetá), una propuesta metodológica. Seminario Internacional de Fiscalidad Ambiental.

Pardo, Y. Y., Andrade, M.C. \& Hermosa, D. (2012). Evaluación Económica de políticas y proyectos: métodos alternativos y estudios de caso. FERIVA, Cali Colombia

Peters, A., Goldstein, I. F., Beyer, U., Franke, K., Heinrich, J., Dockery, D. W., Spengler, J. D., \& Wichmann, H. E. (1996). Acute health effects of exposure to high levels of air pollution in eastern Europe. American journal of epidemiology, 144(6), 570-581.

Sánchez, J., Urrego, J., Zakzuk, J., Bornacelly, A., Castro, I., \& Caraballo, L. (2013). Niveles de Contaminantes en el aire de Cartagena, Colombia. Revista Salud UIS, 45(3).

Simioni, D. (Ed.). (2003). Contaminación atmosférica y conciencia ciudadana (Vol. 73). United Nations Publications.

Wark, K., \& Warmer, C. F. (1994). Contaminación del aire: origen y control. Limusa.

Tyler, C. N., Acevedo, J., Bocarejo, J. P., \& Velásquez, J. M. (2013). Caracterización de la contaminación atmosférica en Colombia.

Uribe, E; Mendieta, J; Rueda, H. \& Carriazo, F. (2003). Introducción a la valoración ambiental y estudios de caso. CEDE - COLCIENCIAS - Ediciones, Uniandes. 